\title{
Nutritional and 13-Week Subchronic Toxicological Evaluation of Lignosus rhinocerotis Mycelium in Sprague-Dawley Rats
}

\author{
I-Chen Li ${ }^{1}$, Bi-Hua Yang ${ }^{1}$, Jing-Yi Lin ${ }^{1}$, Shan Lin $^{1}$ and Chin-Chu Chen ${ }^{1,2,3,4, *}$ \\ 1 Biotech Research Institute, Grape King Bio Ltd., Taoyuan City 325, Taiwan; \\ ichen.li@grapeking.com.tw (I.-C.L.); sybil.yang@grapeking.com.tw (B.-H.Y.); \\ jingyi.lin@grapeking.com.tw (J.-Y.L.); shan.lin@grapeking.com.tw (S.L.) \\ 2 Department of Food Science, Nutrition, and Nutraceutical Biotechnology, Shih Chien University, \\ Taipei City 104, Taiwan \\ 3 Institute of Food Science and Technology, National Taiwan University, Taipei City 106, Taiwan \\ 4 Department of Bioscience Technology, Chung Yuan Christian University, Taoyuan City 320, Taiwan \\ * Correspondence: gkbioeng@grapeking.com.tw
}

check for updates

Citation: Li, I.-C.; Yang, B.-H.; Lin, J.-Y.; Lin, S.; Chen, C.-C. Nutritional and 13-Week Subchronic

Toxicological Evaluation of Lignosus rhinocerotis Mycelium in Sprague-Dawley Rats. Int. J. Environ. Res. Public Health 2021, 18, 1271. https://doi.org/10.3390/ijerph18031271

Academic Editor: Oliver Grundmann Received: 7 January 2021

Accepted: 28 January 2021

Published: 31 January 2021

Publisher's Note: MDPI stays neutral with regard to jurisdictional claims in published maps and institutional affiliations.

Copyright: (C) 2021 by the authors. Licensee MDPI, Basel, Switzerland. This article is an open access article distributed under the terms and conditions of the Creative Commons Attribution (CC BY) license (https:// creativecommons.org/licenses/by/ $4.0 /)$.

\begin{abstract}
Lignosus rhinocerotis (Tiger's Milk mushroom) is a novel mushroom with sclerotium belonging to the Polyporaceae family and has been reported widely to possess anti-cancer, anti-cough, antioxidant, gastro-protective, immuno-modulating, and neurite-stimulating properties. As numerous studies have proven the tremendous medicinal values of L. rhinocerotis, it is necessary to understand its nutrition as well as its safety for the recipient. Previous research on L. rhinocerotis has mainly focused on the naturally occurring sclerotium and may have overlooked mushroom mycelia from submerged liquid fermentation, which ensures a high uniform quantitative biomass production as well as a high biological value. Hence, this is the first report on the evaluation of nutrition and 13-week repeated oral toxicity of L. rhinocerotis mycelium (LRM). The LRM powder contained $9.0 \pm 4.2 \%$ moisture, $1.9 \pm 1.3 \%$ ash, $1.6 \pm 2.2 \%$ crude lipid, $8.4 \pm 5.3 \%$ crude protein, $79.3 \pm 4.6 \%$ carbohydrate, and $364 \mathrm{kcal} / 100 \mathrm{~g}$ energy. The total free amino acid ranged from 349 to $5636 \mathrm{mg} / 100 \mathrm{~g}$ and the umami index of freeze-dried LRM powder was 0.37 . For safety assessment, ninety-six rats were divided into four groups, each consisting of twelve male and twelve female rats. Test articles were administered by oral gavage to rats at 850, 1700, and $3400 \mathrm{mg} / \mathrm{kg}$ body weight/day for 13 weeks and reverse osmosis water was used as the control. All animals survived to the end of the study. During the experiment period, no abnormal changes were observed in clinical signs, body weight, or ophthalmological examinations. No adverse or test article-related differences were found in urinalysis, hematology, or serum biochemistry parameters between the treatment and control groups. Necropsy and histopathological examination indicated no treatment-related changes. According to the above results, the no-observed-adverse-effect level (NOAEL) of L. rhinocerotis was identified to be greater than $3400 \mathrm{mg} / \mathrm{kg}$ body weight (BW)/day in Sprague-Dawley rats.
\end{abstract}

Keywords: Lignosus rhinocerotis; mycelia; umami index; 13 week toxicity; NOAEL

\section{Introduction}

Mushroom fruiting bodies and mycelia have been recognized not only as a part of a regular diet with high nutrient value but also as a general health tonic for various ailments from time immemorial [1]. Extensive research on mushrooms has markedly increased, mainly due to their natural products with various biological activities [2]. They are reputed to exhibit medicinal benefits such as cholesterol-reducing, liver protective, immunemodulating, anti-diabetic, anti-inflammatory, anti-oxidant, anti-tumor, anti-microbial, and anti-viral activities [3]. With these various human health benefits, it is also fundamental to emphasize their toxicity characteristics as well as their safety for use in therapy or in human nutrition. 
Lignosus rhinocerotis (Cooke) Ryvarden, also known as Tiger's Milk mushroom, is one of the most highly valued medicinal mushrooms sought after by the natives of Malaysia [4]. Its underground sclerotium (a compact of hardened fungal mycelium) is the part with medicinal value and has been used traditionally for the treatment of liver cancer, chronic hepatitis, and gastric ulcers [5]. Furthermore, recent research has demonstrated several bioactive properties of the sclerotium of L. rhinocerotis such as the enhancement of immune-modulatory actions on tumor cells [6], stimulatory effects on neurite outgrowth [7], anti-proliferative activities against carcinoma cells [8], and anti-acute inflammatory activities in the carrageenan-induced paw edema test [9]. Hence, there is a possibility of commercializing L. rhinocerotis sclerotium as a potential functional ingredient in food or dietary supplements.

Unfortunately, the supply of L. rhinocerotis sclerotium is limited as its presence can only be noticed when the fruiting body sprouts out from the ground [10]. Therefore, attempts have been made to cultivate this highly prized sclerotium or mycelium on agroresidues [11] or by liquid fermentation [12], respectively. However, the production of sclerotium usually takes several months [13], and it is often difficult to control the quality of the final product. For this reason, the potential of mycelium as a substitute for sclerotium concerning its bioactivities and chemical substitutes has received a great deal of attention. Furthermore, a recent study showed that $L$. rhinocerotis from liquid fermentation merits consideration as a potential substitute for the sclerotium from comparative studies on bioactivity evaluation and chemical profiling [12].

To date, results show that the L. rhinocerotis mycelium is devoid of its genotoxic effect under the experimental conditions [14] and its no-observed-adverse-effect level (NOAEL) is greater than $3400 \mathrm{mg} / \mathrm{kg} \mathrm{BW}$ in reproductive and developmental animal studies using rats [15]. As part of an overall program to evaluate the effect and safety of this promising natural remedy for general use in food or health supplements, it is therefore necessary to carry out in-depth nutrition and safety evaluations. Thus, the present study was conducted to assess the nutritional and amino acid components as well as the 13-week subchronic toxicity in Sprague-Dawley rats, for demonstrating either long-term safety or prediction of acceptable daily intake (ADI). This study was performed in compliance with the testing guidelines of the Organization for Economic Cooperation and Development (OECD) and the US Food and Drug Administration (FDA) Good Laboratory Practice Regulations.

\section{Materials and Methods}

\subsection{L. rhinocerotis Preparation}

Lignosus rhinocerotis fruiting body was purchased from Ligno Biotech at Selangor, Malaysia (voucher code \#0032). Mycelium was isolated and authenticated by sequencing using internal transcribed spacers (ITSs) 1 and 2. After our isolation demonstrated $99 \%$ similarity compared to the biological database using the Basic Local Alignment Search Tool (BLAST) (blast.ncbi.nlm.nih.gov/) (data not shown), the specimen was deposited at Bioresource Collection and Research Centre (BCRC) as BCRC 930202 in Taiwan.

Fresh mycelium was transferred to fresh potato dextrose agar (PDA) plates every 15 days. A size of $1 \mathrm{~cm}^{3}$ was cut and inoculated into a 2-L Hinton flask containing $500 \mathrm{~mL}$ of nutrient medium consisting of $2 \%$ glucose, $0.5 \%$ soya bean powder, $0.2 \%$ peptone, and $0.1 \%$ yeast extract, with $\mathrm{pH}$ adjusted to 5.0 with $0.1 \mathrm{M}$ hydrochloric acid. The flask was cultured at $100 \mathrm{rpm}$ and incubated at $25^{\circ} \mathrm{C}$ for 5 days before being transferred to a 500-L pilot seed fermenter containing $350 \mathrm{~L}$ of the same medium. After 4 days, this seed was transferred to a 50-ton full-scale fermenter and cultured under the same conditions. Mature mycelia were separated from the culture broth, lyophilized, ground to a powder, and stored at $-20{ }^{\circ} \mathrm{C}$.

\subsection{Proximate Analysis, Amino Acid Composition, and Umami Index}

The proximate composition analysis, including crude protein, total crude lipid, ash, fiber, and moisture contents, of the freeze-dried mycelia was determined according to The 
Association of Official Analytical Chemists (AOAC) official methods 984.13, 43.275, 968.08, 991.43, and 950.46. B, respectively [16]. The energy values of the samples were determined by multiplying the protein content by 4 , carbohydrate content by 4 , and fat content by 9 . The analysis of amino acid content was analyzed based on AOAC 994.12. The mushroom taste, also called the umami or palatable index, was determined using the ratio of aspartic and glutamic acids over the total amino acids according to a previous method [17].

\subsection{Animals}

Forty-eight male and 48 female, six-week-old, Sprague-Dawley rats (BioLASCO, Taipei, Taiwan) were acclimated for 8 days before being randomly assigned to the control and three treatment groups of 12 rats per sex in each group. The body weight (BW) of the rats ranged from 196.2 to $236.9 \mathrm{~g}$ and from 145.7 to $173.4 \mathrm{~g}$ for the males and females, respectively. All animals were identified by an ear notch and were housed in pairs maintained under a relative humidity $(55 \pm 20 \%)$, controlled temperature $\left(21 \pm 2{ }^{\circ} \mathrm{C}\right)$, with alternating light cycles (12 h of light/dark). The frequency of ventilation was 10-15 times/h. During the study period, the animals had free ad libitum access to standard rodent diet (Laboratory Autoclavable Rodent Diet ${ }^{\circledR}$ \# 5010, PMI Nutrition International, Brentwood, MO, USA) and autoclaved, reverse-osmosis water. Aspen Chip bedding (Northeastern Products, Bellmore, NY, USA) was changed weekly. BW of all rats were measured before administration of the test article. All animal procedures were reviewed and approved by the Institutional Animal Care and Use Committee (IACUC, New Taipei City, Taiwan) at Level Laboratory Animal Center in Taiwan (approval No.: 120201-04).

\subsection{Study Design}

This study was performed based on the Organization for Economic Co-operation and Development (OECD) Guideline 408 [18] and was in strict accordance with the Good Laboratory Practice for Non-Clinical Laboratory Studies (FDA, 21 CFR, Part 58), Good Laboratory Practice for Non-Clinical Laboratory Studies (DOH, R.O.C., 3rd ed., 2006) and OECD Principles of Good Laboratory Practice (TAF OECD GLP Compliance, No. 1, 1997). The BWs of all rats were measured weekly until a scheduled necropsy 90 days later. Feed and water consumption were recorded weekly during the study period. Lignosus rhinocerotis mycelia (LRM) powder was given to rats daily by oral gavage at different dosages: $0 \mathrm{mg} / \mathrm{kg} \mathrm{BW} /$ day (control), $850 \mathrm{mg} / \mathrm{kg} \mathrm{BW} /$ day (low), $1700 \mathrm{mg} / \mathrm{kg}$ BW/day (medium), and $3400 \mathrm{mg} / \mathrm{kg} \mathrm{BW/day} \mathrm{(high).} \mathrm{LRM} \mathrm{was} \mathrm{prepared} \mathrm{fresh} \mathrm{daily} \mathrm{and}$ administered to each rat via a stainless-steel ball-tipped gavage needle. Clinical observations of mortality, morbidity, and possible signs of toxicity were made daily during the experimental period. At the end of the experiment, all surviving animals were anesthetized with a mixed solution of ketamine $(80 \mathrm{mg} / \mathrm{mL})$ and xylazine $(8 \mathrm{mg} / \mathrm{mL})$, followed by blood collection, exsanguination, and a necropsy. Gross necropsies included an examination of the external surface of the body, all thoracic and abdominal cavities, intestines, and visceral organs.

\subsection{Urinalysis}

Before the gross necropsy examination, urine samples were collected approximately 12 $16 \mathrm{~h}$ prior to terminal sacrifice using metabolism cages. Immediately after each urine sample was obtained, the $\mathrm{pH}$, specific gravity, protein, glucose, ketone, bilirubin, urobilinogen, occult blood, leukocytes, and nitrite were analyzed with a urine analyzer (Bayer ClinitekStatus, Siemens, Malvern, PA, USA). Urine sediments were observed for red blood cells (RBCs), white blood cells (WBCs), cells, casts, crystals, and microbes using a Nikon E200 microscope (Tokyo, Japan).

\subsection{Hematology and Serum Biochemistry}

Hematology and serum chemistry were performed after the 13-week dosing period. On the necropsy day, blood samples were obtained through the abdominal aorta and 
collected into three tubes that contained: (1) K2 EDTA for complete blood count analysis; (2) sodium citrate for a coagulation factor analysis; and (3) no anticoagulant for serum chemical analysis. Hematology examinations were performed using an automatic blood analyzer (Sysmex Xt-1800i, Sysmex, Kobe, Japan), which included analysis of the red blood cells (RBCs), white blood cells (WBCs), platelets, hemoglobin, hematocrit, mean corpuscular volume (MCV), mean corpuscular hemoglobin $(\mathrm{MCH})$, mean corpuscular hemoglobin concentration (MCHC), neutrophils, eosinophils, basophils, monocytes, and lymphocytes. Anticoagulated blood samples were analyzed by blood coagulation analyzer (Sysmex CA-540, Siemens, Malvern, PA, USA) for the prothrombin time and activated partial thromboplastin time (APTT). An automated assay was performed with a model 7080 Hitachi analyzer (Tokyo, Japan) to test the following clinical chemical parameters: amylase, albumin, alkaline phosphatase (ALP), total bilirubin, alanine aminotransferase (ALT), aspartate aminotransferase (AST), gamma-glutamyl transferase (GGT), total protein, creatinine, blood urea nitrogen (BUN), cholesterol, triglycerides, creatine kinase, chloride, sodium, potassium, glucose, calcium, and phosphorus.

\subsection{Histopathology}

The gross necropsy included examination of the external surface of the body, all thoracic and abdominal cavities, intestines, and visceral organs. The following tissues/organs were collected and weighed wet in situ after dissection: adrenals (paired), brain, epididymides (paired), heart, kidneys (paired), liver, ovaries with oviducts (paired), pituitary, spleen, testes (paired), thymus, and uterus with the cervix. The prostate and seminal vesicles with coagulating glands as a whole were weighed after fixation. Following the gross necropsy, these tissues/organs were fixed and preserved in 10\% neutral buffered formalin or other appropriate fixatives for subsequent histopathological examination. Selected tissues in the control group and high dose group were trimmed, embedded, sectioned, and H\&E-stained and then underwent a microscopic (Nikon E200, Tokyo, Japan) examination, according to a previous study [19].

\subsection{Statistical Analysis}

Data collected from treated and control groups were compared by the one-way analysis of variance (ANOVA) method, followed by Dunnett's method (SPSS, vers. 12.0, Illinois, Chicago, USA). A probability of $0.05(p<0.05)$ was used as the criterion of significance.

\section{Results}

\subsection{Proximate and Amino Acid Analysis of LRM Powder}

The result of proximate analysis of freeze-dried LRM powder is shown in Table 1. The LRM powder contained $9.0 \pm 4.2 \%$ moisture, $1.9 \pm 1.3 \%$ ash, $1.6 \pm 2.2 \%$ crude lipid, $8.4 \pm 5.3 \%$ crude protein, $79.3 \pm 4.6 \%$ carbohydrate, and $364 \mathrm{kcal} / 100 \mathrm{~g}$ energy. The total free amino acid ranged from 349 to $5636 \mathrm{mg} / 100 \mathrm{~g}$ and the umami index of freeze-dried LRM powder was 0.37 (Table 2).

Table 1. Proximate composition of L. rhinocerotis mycelium (LRM) powder.

\begin{tabular}{cc}
\hline & Proximate Analysis (\%) \\
\hline Moisture & $9.0 \pm 4.2$ \\
Ash & $1.9 \pm 1.3$ \\
Crude lipid & $1.6 \pm 2.2$ \\
Crude protein & $8.4 \pm 5.3$ \\
Carbohydrate & $79.3 \pm 4.6$ \\
Energy (kcal/100 g) & $364.0 \pm 22.6$ \\
\hline
\end{tabular}

Each value is expressed in mean $\pm \mathrm{SD}(n=2$.). 
Table 2. The amino acid composition and the umami index of freeze-dried LRM powder.

\begin{tabular}{cc}
\hline Amino Acid & $\mathbf{m g} \mathbf{1 0 0} \mathbf{g}$ \\
\hline Aspartate & 2384 \\
Glutamate & 5636 \\
Serine & 1007 \\
Histidine & 588 \\
Glycine & 1106 \\
Threonine & 1054 \\
Alanine & 1218 \\
Arginine & 1766 \\
Tyrosine & 436 \\
Cystine & 349 \\
Valine & 753 \\
Methionine & 360 \\
Phenylalanine & 718 \\
Isoleucine & 764 \\
Leucine & 1205 \\
Lysine & 1355 \\
Proline & 1030 \\
\hline Umami index ${ }^{\text {a }}$ & 0.37
\end{tabular}

a Ratio of aspartic and glutamic acids to total amino acids, calculated according to a previous method [17].

\subsection{Effect of LRM Powder on Body Weight and Feed Intake}

Oral administration of LRM powder via gavage for 13 consecutive weeks showed no mortality or toxicity symptoms in any rats. Regarding body weight, there were no significant differences in any treated groups (Figure 1). There were also no significant differences among groups regarding feed intake except for male rats administered $3400 \mathrm{mg} / \mathrm{kg} /$ day LRM $(p<0.05)$ (Figure 2). This reduction was slight, reduced only in males, and was therefore considered to be an accidental change.

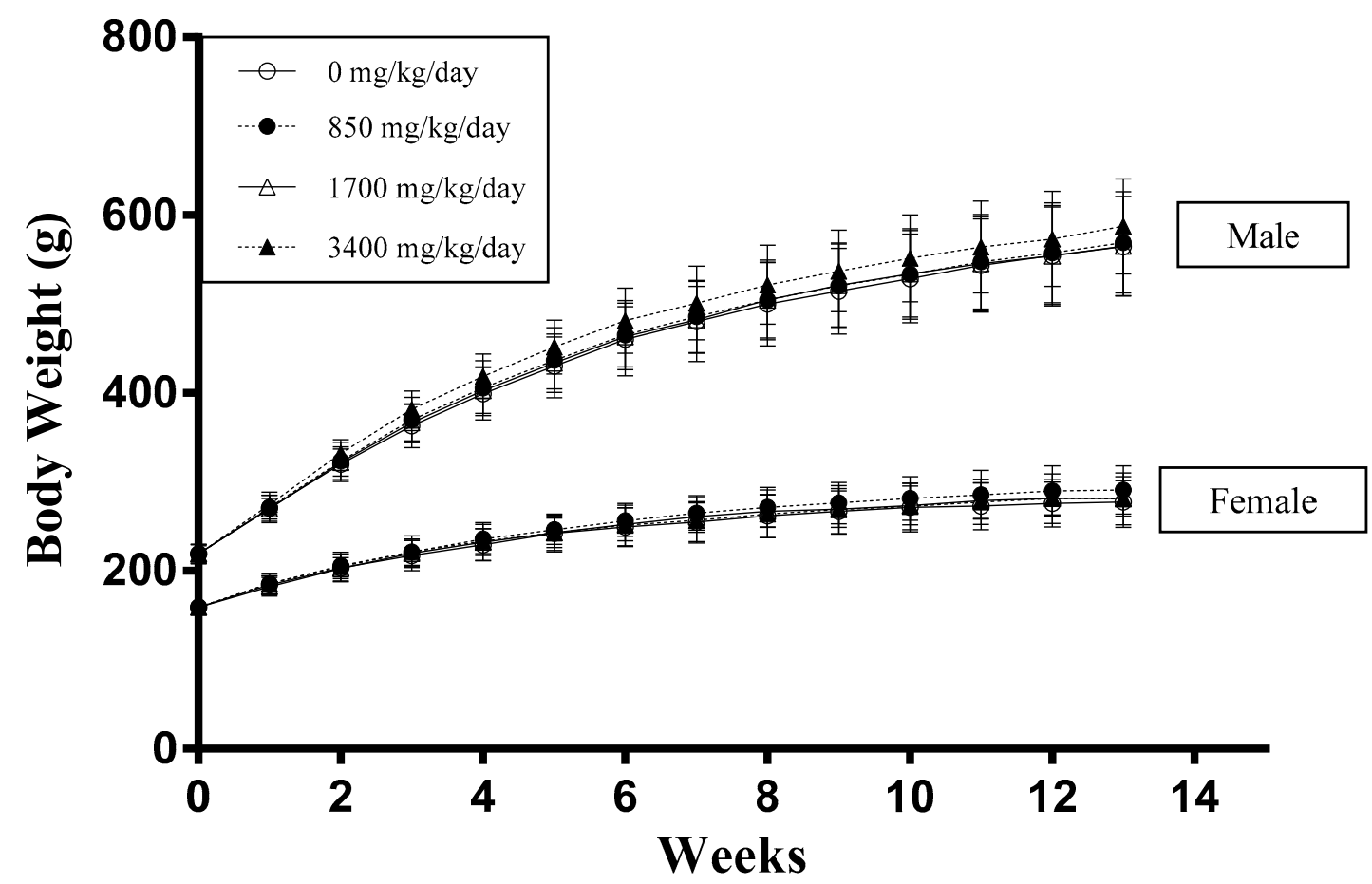

Figure 1. Effect of LRM powder on body weight of male and female Sprague-Dawley rats. Data expressed as mean $\pm \mathrm{SD}$ $(n=12)$. 


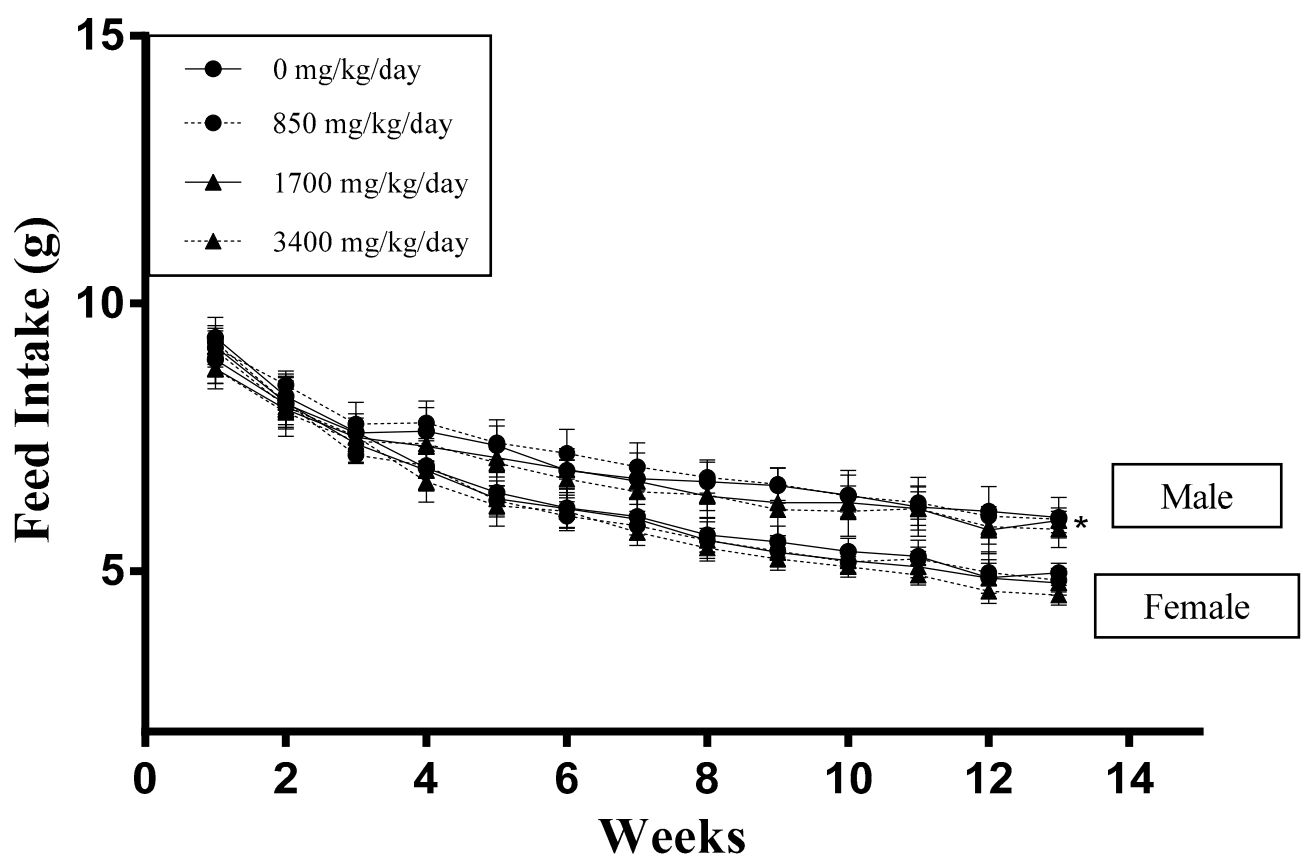

Figure 2. Effect of LRM powder on feed intake of male and female Sprague-Dawley rats. Data expressed as mean $\pm \mathrm{SD}$ $(n=12)$.

\subsection{Effect of LRM Powder in Hematological Parameters and Clinical Biochemistry}

The summary of hematology results is presented in Table 3. No statistically significant changes were detected in the control and treated rats at all the doses tested. In addition, all clinical biochemistry remained unaltered in rats fed with LRM powder when compared to that of the untreated male and female rats (Table 4).

Table 3. Effects of LRM powder on hematology in male and female Sprague-Dawley rats after 13 weeks.

\begin{tabular}{|c|c|c|c|c|}
\hline \multirow{2}{*}{ Parameters } & \multirow{2}{*}{$\begin{array}{c}\text { Control (Distilled } \\
\text { Water) }\end{array}$} & \multicolumn{3}{|c|}{ Lignosus rhinocerotis Mycelium (mg/kg) } \\
\hline & & 850 & 1700 & 3400 \\
\hline \multicolumn{5}{|l|}{ Male } \\
\hline $\operatorname{WBC}\left(10^{3} / \mu \mathrm{L}\right)$ & $5.648 \pm 1.007$ & $6.916 \pm 2.754$ & $7.327 \pm 1.820$ & $6.308 \pm 1.258$ \\
\hline $\operatorname{RBC}\left(10^{6} / \mu \mathrm{L}\right)$ & $9.288 \pm 0.383$ & $9.343 \pm 0.412$ & $9.464 \pm 0.360$ & $9.173 \pm 0.363$ \\
\hline Hemoglobin $(\mathrm{g} / \mathrm{dL})$ & $16.00 \pm 0.44$ & $16.26 \pm 0.54$ & $16.36 \pm 0.63$ & $15.93 \pm 0.51$ \\
\hline Hematocrit (\%) & $44.69 \pm 1.16$ & $45.41 \pm 1.32$ & $45.47 \pm 1.63$ & $44.67 \pm 1.68$ \\
\hline $\mathrm{MCV}(\mathrm{fL})$ & $48.18 \pm 1.87$ & $48.64 \pm 1.56$ & $48.08 \pm 1.89$ & $48.75 \pm 2.09$ \\
\hline $\mathrm{MCH}(\mathrm{pg})$ & $17.25 \pm 0.63$ & $17.44 \pm 0.49$ & $17.29 \pm 0.55$ & $17.38 \pm 0.58$ \\
\hline $\mathrm{MCHC}(\mathrm{g} / \mathrm{dL})$ & $35.79 \pm 0.48$ & $35.80 \pm 0.29$ & $35.98 \pm 0.64$ & $35.68 \pm 0.66$ \\
\hline Platelet $\left(10^{3} / \mu \mathrm{L}\right)$ & $1136.8 \pm 109.6$ & $1169.5 \pm 79.9$ & $1174.2 \pm 151.6$ & $1189.7 \pm 154.5$ \\
\hline Neutrophil (\%) & $29.41 \pm 8.18$ & $31.87 \pm 5.91$ & $34.77 \pm 9.05$ & $30.28 \pm 10.78$ \\
\hline Lymphocyte (\%) & $65.73 \pm 9.12$ & $63.24 \pm 6.09$ & $60.37 \pm 9.33$ & $64.21 \pm 11.16$ \\
\hline Monocyte (\%) & $4.59 \pm 1.61$ & $4.62 \pm 0.89$ & $4.66 \pm 0.82$ & $5.18 \pm 1.45$ \\
\hline Eosinophil (\%) & $0.28 \pm 0.28$ & $0.26 \pm 0.15$ & $0.20 \pm 0.13$ & $0.34 \pm 0.30$ \\
\hline Basophil (\%) & $0.00 \pm 0.00$ & $0.02 \pm 0.04$ & $0.01 \pm 0.03$ & $0.00 \pm 0.00$ \\
\hline PT (sec) & $11.17 \pm 1.34$ & $12.02 \pm 2.28$ & $11.88 \pm 1.83$ & $12.54 \pm 2.02$ \\
\hline APTT (sec) & $16.88 \pm 1.40$ & $17.28 \pm 1.78$ & $17.36 \pm 1.07$ & $17.28 \pm 1.19$ \\
\hline \multicolumn{5}{|l|}{ Female } \\
\hline $\operatorname{WBC}\left(10^{3} / \mu \mathrm{L}\right)$ & $4.893 \pm 1.842$ & $5.020 \pm 2.372$ & $6.608 \pm 2.365$ & $6.259 \pm 3.018$ \\
\hline $\operatorname{RBC}\left(10^{6} / \mu \mathrm{L}\right)$ & $8.277 \pm 0.482$ & $8.466 \pm 0.403$ & $8.501 \pm 0.355$ & $8.501 \pm 0.294$ \\
\hline Hemoglobin (g/dL) & $15.20 \pm 0.29$ & $15.45 \pm 0.58$ & $15.61 \pm 0.62$ & $15.50 \pm 0.59$ \\
\hline Hematocrit $(\%)$ & $42.71 \pm 0.41$ & $43.49 \pm 1.52$ & $43.94 \pm 1.74$ & $43.46 \pm 1.35$ \\
\hline $\mathrm{MCV}(\mathrm{fL})$ & $51.75 \pm 2.82$ & $51.40 \pm 1.28$ & $51.73 \pm 1.88$ & $51.15 \pm 1.49$ \\
\hline $\mathrm{MCH}(\mathrm{pg})$ & $18.40 \pm 0.74$ & $18.25 \pm 0.35$ & $18.37 \pm 0.51$ & $18.23 \pm 0.37$ \\
\hline $\mathrm{MCHC}(\mathrm{g} / \mathrm{dL})$ & $35.58 \pm 0.59$ & $35.52 \pm 0.41$ & $35.53 \pm 0.50$ & $35.65 \pm 0.62$ \\
\hline
\end{tabular}


Table 3. Cont.

\begin{tabular}{ccccc}
\hline \multirow{2}{*}{ Parameters } & \multirow{2}{*}{$\begin{array}{c}\text { Control (Distilled } \\
\text { Water) }\end{array}$} & \multicolumn{3}{c}{ Lignosus rhinocerotis Mycelium (mg/kg) } \\
\cline { 3 - 5 } & $1061.5 \pm 89.1$ & $1066.1 \pm 118.4$ & $\mathbf{1 7 0 0}$ & $\mathbf{3 4 0 0}$ \\
\hline Platelet $\left(10^{3} / \mu \mathrm{L}\right)$ & $16.51 \pm 3.56$ & $15.43 \pm 4.20$ & $1065.9 \pm 134.7$ & $1110.2 \pm 85.1$ \\
Neutrophil $(\%)$ & $79.40 \pm 4.06$ & $80.83 \pm 4.51$ & $79.27 \pm 6.50$ & $19.76 \pm 10.38$ \\
Lymphocyte $(\%)$ & $3.83 \pm 1.27$ & $3.48 \pm 1.09$ & $3.73 \pm 0.81$ & $76.02 \pm 10.83$ \\
Monocyte $(\%)$ & $0.27 \pm 0.29$ & $0.28 \pm 0.22$ & $0.23 \pm 0.14$ & $3.98 \pm 0.86$ \\
Eosinophil (\%) & $0.00 \pm 0.00$ & $0.00 \pm 0.00$ & $0.02 \pm 0.04$ & $0.23 \pm 0.28$ \\
Basophil (\%) & $9.34 \pm 0.24$ & $9.36 \pm 0.28$ & $9.22 \pm 0.26$ & $0.02 \pm 0.06$ \\
Reticulocyte (\%) & $14.71 \pm 0.95$ & $14.45 \pm 0.83$ & $14.72 \pm 0.81$ & $9.36 \pm 0.15$ \\
APTT (sec) & & & $15.13 \pm 0.90$ \\
\hline
\end{tabular}

Data were expressed as mean \pm SD ( $n=12$ ). White blood cell (WBC); Red blood cell (RBC); Mean corpuscular volume (MCV); Mean corpuscular hemoglobin (MCH); Mean corpuscular hemoglobin concentration (MCHC); Activated partial thromboplastin time (APTT).

Table 4. Effects of LRM powder on clinical biochemistry in male and female Sprague-Dawley rats after 13 weeks.

\begin{tabular}{|c|c|c|c|c|}
\hline \multirow{2}{*}{ Parameters } & \multirow{2}{*}{ Control (Distilled Water) } & \multicolumn{3}{|c|}{ Lignosus rhinocerotis Mycelium (mg/kg) } \\
\hline & & 850 & 1700 & 3400 \\
\hline \multicolumn{5}{|l|}{ Male } \\
\hline AST (U/L) & $117.96 \pm 22.63$ & $122.56 \pm 17.88$ & $127.61 \pm 26.56$ & $129.73 \pm 16.56$ \\
\hline ALT $(\mathrm{U} / \mathrm{L})$ & $32.86 \pm 5.69$ & $30.06 \pm 4.30$ & $29.24 \pm 4.41$ & $31.93 \pm 11.01$ \\
\hline Glucose (mg/dL) & $152.68 \pm 19.85$ & $167.53 \pm 42.16$ & $154.78 \pm 22.24$ & $160.41 \pm 19.35$ \\
\hline Total protein $(\mathrm{g} / \mathrm{dL})$ & $6.13 \pm 0.32$ & $6.14 \pm 0.36$ & $6.09 \pm 0.37$ & $6.01 \pm 0.40$ \\
\hline Albumin $(\mathrm{g} / \mathrm{dL})$ & $4.06 \pm 0.22$ & $3.98 \pm 0.23$ & $3.99 \pm 0.34$ & $3.94 \pm 0.25$ \\
\hline Total bilirubin (mg/dL) & $<0.04$ & $<0.04$ & $<0.04$ & 0.05 \\
\hline $\mathrm{BUN}(\mathrm{mg} / \mathrm{dL})$ & $15.36 \pm 1.80$ & $15.49 \pm 2.09$ & $14.16 \pm 2.70$ & $14.86 \pm 2.09$ \\
\hline Creatinine (mg/dL) & $0.50 \pm 0.04$ & $0.51 \pm 0.09$ & $0.46 \pm 0.07$ & $0.54 \pm 0.11$ \\
\hline GGT $(\mathrm{U} / \mathrm{L})$ & $<2.0$ & $<2.0$ & $<2.0$ & $<2.0$ \\
\hline Alkaline phosphatase (U/L) & $256.92 \pm 68.56$ & $265.83 \pm 57.40$ & $0.63 \pm 55.61$ & $236.48 \pm 46.79$ \\
\hline Cholesterol (mg/dL) & $61.32 \pm 14.20$ & $64.13 \pm 14.50$ & $49.78 \pm 9.82$ & $53.70 \pm 11.30$ \\
\hline Triglyceride (mg/dL) & $20.43 \pm 5.69$ & $26.18 \pm 15.45$ & $23.39 \pm 14.68$ & $23.13 \pm 7.58$ \\
\hline Calcium (mg/dL) & $9.49 \pm 0.52$ & $9.63 \pm 0.79$ & $9.53 \pm 0.78$ & $9.34 \pm 0.57$ \\
\hline Phosphorus (mg/dL) & $6.74 \pm 1.06$ & $7.11 \pm 1.10$ & $6.73 \pm 0.92$ & $6.43 \pm 0.92$ \\
\hline Creatine kinase (U/L) & $428.93 \pm 173.14$ & $483.89 \pm 165.51$ & $382.94 \pm 168.04$ & $414.63 \pm 182.19$ \\
\hline Amylase $(\mathrm{U} / \mathrm{L})$ & $1306.3 \pm 180.20$ & $1325.80 \pm 240.70$ & $1391.80 \pm 196.20$ & $1329.30 \pm 164.10$ \\
\hline Sodium (mmol/L) & $145.68 \pm 6.03$ & $143.60 \pm 5.92$ & $144.63 \pm 9.87$ & $144.28 \pm 5.00$ \\
\hline Potassium (mmol/L) & $4.38 \pm 0.20$ & $4.41 \pm 0.25$ & $4.40 \pm 0.35$ & $4.58 \pm 0.27$ \\
\hline Chloride (mmol/L) & $101.29 \pm 4.99$ & $99.66 \pm 5.27$ & $100.95 \pm 8.08$ & $100.53 \pm 3.81$ \\
\hline \multicolumn{5}{|l|}{ Female } \\
\hline AST (U/L) & $80.74 \pm 9.98$ & $97.22 \pm 28.79$ & $84.07 \pm 17.49$ & $85.07 \pm 23.27$ \\
\hline ALT $(\mathrm{U} / \mathrm{L})$ & $19.91 \pm 5.28$ & $23.31 \pm 6.43$ & $19.11 \pm 3.41$ & $34.12 \pm 53.87$ \\
\hline Glucose (mg/dL) & $126.01 \pm 24.32$ & $137.93 \pm 31.11$ & $124.50 \pm 27.44$ & $124.53 \pm 24.10$ \\
\hline Total protein (g/dL) & $5.88 \pm 0.68$ & $6.38 \pm 0.89$ & $5.91 \pm 0.95$ & $5.99 \pm 0.83$ \\
\hline Albumin (g/dL) & $4.14 \pm 0.47$ & $4.48 \pm 0.63$ & $4.12 \pm 0.52$ & $4.22 \pm 0.54$ \\
\hline Total bilirubin (mg/dL) & $0.07 \pm 0.02$ & $0.07 \pm 0.03$ & $0.08 \pm 0.03$ & $0.06 \pm 0.01$ \\
\hline $\mathrm{BUN}(\mathrm{mg} / \mathrm{dL})$ & $14.62 \pm 3.12$ & $15.13 \pm 3.15$ & $14.30 \pm 1.78$ & $12.69 \pm 2.40$ \\
\hline Creatinine (mg/dL) & $0.52 \pm 0.07$ & $0.57 \pm 0.16$ & $0.50 \pm 0.06$ & $0.47 \pm 0.10$ \\
\hline GGT $(\mathrm{U} / \mathrm{L})$ & $<2.0$ & $<2.0$ & $<2.0$ & $<2.0$ \\
\hline Alkaline phosphatase (U/L) & $118.96 \pm 43.98$ & $128.16 \pm 43.78$ & $110.61 \pm 38.00$ & $122.11 \pm 28.83$ \\
\hline Cholesterol (mg/dL) & $53.69 \pm 16.20$ & $60.08 \pm 19.43$ & $59.78 \pm 11.48$ & $54.46 \pm 18.70$ \\
\hline Triglyceride (mg/dL) & $17.86 \pm 8.31$ & $23.94 \pm 15.83$ & $14.71 \pm 4.72$ & $19.44 \pm 14.77$ \\
\hline Calcium (mg/dL) & $9.03 \pm 0.82$ & $9.68 \pm 1.41$ & $8.87 \pm 1.26$ & $8.90 \pm 1.08$ \\
\hline Phosphorus (mg/dL) & $5.78 \pm 1.02$ & $5.85 \pm 2.46$ & $5.58 \pm 0.90$ & $5.20 \pm 0.78$ \\
\hline Creatine kinase (U/L) & $301.18 \pm 79.02$ & $397.21 \pm 179.35$ & $338.78 \pm 171.80$ & $332.68 \pm 196.74$ \\
\hline Amylase $(\mathrm{U} / \mathrm{L})$ & $849.70 \pm 169.90$ & $1028.80 \pm 314.60$ & $852.70 \pm 240.50$ & $815.10 \pm 149.50$ \\
\hline Sodium $(\mathrm{mmol} / \mathrm{L})$ & $133.42 \pm 13.66$ & $142.69 \pm 16.82$ & $134.54 \pm 14.85$ & $134.18 \pm 12.54$ \\
\hline Potassium (mmol/L) & $4.35 \pm 0.90$ & $4.80 \pm 1.96$ & $4.04 \pm 0.57$ & $4.09 \pm 0.43$ \\
\hline Chloride (mmol/L) & $97.38 \pm 11.93$ & $104.09 \pm 13.38$ & $97.19 \pm 11.12$ & $97.61 \pm 9.54$ \\
\hline
\end{tabular}

Data were expressed as mean $\pm \mathrm{SD}(n=12)$. Aspartate aminotransferase (AST); Alanine aminotransferase (ALT); Alkaline phosphatase (ALP); Gamma-glutamyl transferase (GGT); Blood urea nitrogen (BUN). 


\subsection{Effect of LRM Powder in Urinalysis}

All urinary parameters in all groups were comparable among all the groups at the scheduled analysis, and there were no statistically or biologically significant changes between the treatment groups and the control group (Table 5).

Table 5. Effects of L. rhinocerotis mycelium on urinalysis parameters of male and female Sprague-Dawley rats after 13 weeks.

\begin{tabular}{ccccc}
\hline Parameters & $\begin{array}{c}\text { Control (Distilled } \\
\text { Water) }\end{array}$ & \multicolumn{3}{c}{ Lignosus rhinocerotis Mycelium (mg/kg) } \\
\cline { 3 - 5 } & & $\mathbf{8 5 0}$ & $\mathbf{1 7 0 0}$ & $\mathbf{3 4 0 0}$ \\
\hline Male & $19.08 \pm 8.16$ & $17.46 \pm 8.18$ & $19.46 \pm 10.57$ & $16.79 \pm 6.08$ \\
Volume (mL) & $1.017 \pm 0.004$ & $1.015 \pm 0.003$ & $1.018 \pm 0.004$ & $1.019 \pm 0.004$ \\
Specific Gravity & $7.29 \pm 0.45$ & $7.08 \pm 0.19$ & $7.13 \pm 0.23$ & $7.00 \pm 0.30$ \\
pH & $0.20 \pm 0.00$ & $0.20 \pm 0.00$ & $0.20 \pm 0.00$ & $0.20 \pm 0.00$ \\
Urobilinogen (EU/dL) & & & & $10.83 \pm 4.74$ \\
Female & $12.92 \pm 8.27$ & $12.63 \pm 5.53$ & $1.018 \pm 0.003$ & $1.016 \pm 0.004$ \\
Volume (mL) & $1.016 \pm 0.004$ & $1.017 \pm 0.004$ & $6.92 \pm 0.19$ & $7.00 \pm 0.37$ \\
Specific Gravity & $7.00 \pm 0.21$ & $6.96 \pm 0.14$ & $0.20 \pm 0.00$ & $0.27 \pm 0.23$ \\
pH & $0.33 \pm 0.31$ & $0.20 \pm 0.00$ &
\end{tabular}

Data were expressed as mean $\pm \mathrm{SD}(n=12)$.

\subsection{Effect of LRM Powder in Necropsy, Organ Weight, and Histopathology}

Necropsy and macroscopic examination of organs and tissues from rats sub-chronically exposed to LRM showed no remarkable treatment-related damage. Moreover, no significant differences in the absolute weights of the measured organs were observed between any LRM-treated group and the concurrent control group for both sexes except an increased ovary with oviducts weight of female rats noted in the $850 \mathrm{mg} / \mathrm{kg}$ of LRM treatment groups at the end of the treatment period (Table 6). However, such differences were spontaneous, non-dose-dependent, and therefore not considered toxicologically or biologically relevant.

Table 6. Effects of L. rhinocerotis mycelium on organ weight of male and female Sprague-Dawley rats after 13 weeks.

\begin{tabular}{|c|c|c|c|c|}
\hline \multirow{2}{*}{ Parameters } & \multirow{2}{*}{$\begin{array}{c}\text { Control (Distilled } \\
\text { Water) }\end{array}$} & \multicolumn{3}{|c|}{ Lignosus rhinocerotis Mycelium (mg/kg) } \\
\hline & & 850 & 1700 & 3400 \\
\hline \multicolumn{5}{|l|}{ Male } \\
\hline Adrenals (Paired) & $0.053 \pm 0.005$ & $0.058 \pm 0.008$ & $0.054 \pm 0.009$ & $0.057 \pm 0.007$ \\
\hline Brain & $2.177 \pm 0.091$ & $2.158 \pm 0.069$ & $2.183 \pm 0.058$ & $2.113 \pm 0.087$ \\
\hline Pituitary & $0.014 \pm 0.002$ & $0.013 \pm 0.001$ & $0.013 \pm 0.001$ & $0.013 \pm 0.001$ \\
\hline Thymus & $0.421 \pm 0.122$ & $0.389 \pm 0.081$ & $0.421 \pm 0.099$ & $0.383 \pm 0.108$ \\
\hline Heart & $1.583 \pm 0.152$ & $1.575 \pm 0.138$ & $1.538 \pm 0.110$ & $1.705 \pm 0.149$ \\
\hline Kidney (Paired) & $3.468 \pm 0.389$ & $3.468 \pm 0.364$ & $3.588 \pm 0.341$ & $3.519 \pm 0.458$ \\
\hline Liver & $14.519 \pm 1.988$ & $14.548 \pm 2.409$ & $14.750 \pm 1.633$ & $15.286 \pm 2.676$ \\
\hline Spleen & $0.860 \pm 0.195$ & $0.918 \pm 0.213$ & $0.815 \pm 0.174$ & $0.854 \pm 0.165$ \\
\hline Testes (Paired) & $3.493 \pm 0.258$ & $3.370 \pm 0.355$ & $3.281 \pm 0.140$ & $3.214 \pm 0.376$ \\
\hline Epididymides (Paired) & $1.448 \pm 0.172$ & $1.374 \pm 0.126$ & $1.434 \pm 0.060$ & $1.393 \pm 0.129$ \\
\hline $\begin{array}{l}\text { Prostates and seminal } \\
\text { vesicles with } \\
\text { coagulating glands } \\
\text { Female }\end{array}$ & $3.803 \pm 0.427$ & $3.600 \pm 0.321$ & $3.580 \pm 0.449$ & $3.630 \pm 0.394$ \\
\hline Adrenals (Paired) & $0.069 \pm 0.016$ & $0.068 \pm 0.013$ & $0.065 \pm 0.011$ & $0.070 \pm 0.009$ \\
\hline Brain & $1.939 \pm 0.064$ & $1.995 \pm 0.079$ & $1.911 \pm 0.108$ & $1.945 \pm 0.080$ \\
\hline Pituitary & $0.016 \pm 0.003$ & $0.015 \pm 0.002$ & $0.015 \pm 0.003$ & $0.015 \pm 0.003$ \\
\hline Thymus & $0.256 \pm 0.061$ & $0.291 \pm 0.050$ & $0.285 \pm 0.070$ & $0.301 \pm 0.075$ \\
\hline Heart & $0.898 \pm 0.089$ & $0.909 \pm 0.053$ & $0.874 \pm 0.092$ & $0.912 \pm 0.060$ \\
\hline Kidney (Paired) & $1.845 \pm 0.337$ & $1.881 \pm 0.163$ & $1.846 \pm 0.192$ & $1.903 \pm 0.192$ \\
\hline Liver & $7.303 \pm 0.762$ & $7.703 \pm 0.658$ & $7.193 \pm 0.777$ & $7.486 \pm 0.536$ \\
\hline Spleen & $0.487 \pm 0.077$ & $0.507 \pm 0.075$ & $0.529 \pm 0.106$ & $0.524 \pm 0.104$ \\
\hline Ovaries with oviducts & $0.116 \pm 0.013$ & $0.142 \pm 0.0247$ * & $0.135 \pm 0.027$ & $0.136 \pm 0.018$ \\
\hline Uterus with cervix & $0.817 \pm 0.318$ & $0.676 \pm 0.227$ & $0.639 \pm 0.163$ & $0.696 \pm 0.249$ \\
\hline
\end{tabular}


The histopathological lesions of the highest dose and control groups included minimal focal round cell collection in stromal cells of the harderian glands, focal minimal cysts in the ultimobranchial of the thyroid, focal minimal-to-mild myocarditis in the heart, minimal focal round cell collection in stromal cells of the pancreas, minimal multifocal clear cells in the liver, minimal focal round cell collection in the cortex of the kidneys, minimal-to-mild focal round cell collection in the stromal cells of the prostate, and minimal focal round cell collection in the ovaries and oviduct (Table 7). However, considering the incidence and degree of their occurrence, they were considered to be the frequently spontaneous pathological changes in this strain of this age, and were not related to the administration of LRM.

Table 7. Histological findings.

\begin{tabular}{|c|c|c|c|c|c|}
\hline \multirow{2}{*}{$\begin{array}{c}\text { Parameters } \\
\text { Gender }\end{array}$} & & \multicolumn{2}{|c|}{ Control (Distilled Water) } & \multicolumn{2}{|c|}{$\begin{array}{c}\text { Lignosus rhinocerotis Mycelium } \\
\text { (High Dose) }\end{array}$} \\
\hline & & Male & Female & Male & Female \\
\hline $\begin{array}{l}\text { Histopathologic } \\
\text { Findings }\end{array}$ & Severity $^{a}$ & \multicolumn{4}{|c|}{$(\mathrm{N} / \mathrm{N})^{b}$} \\
\hline \multicolumn{6}{|l|}{ Harderian glands } \\
\hline $\begin{array}{l}\text { Focal round cell } \\
\text { collection } \\
\text { Thyroid }\end{array}$ & 1 & $0 / 12$ & $2 / 12$ & $1 / 12$ & $0 / 12$ \\
\hline $\begin{array}{l}\text { Cyst, } \\
\text { ultimobranchial, } \\
\text { focal } \\
\text { Lung }\end{array}$ & 1 & $1 / 12$ & $2 / 12$ & $2 / 12$ & $1 / 12$ \\
\hline $\begin{array}{l}\text { Alveolar } \\
\text { histiocytosis, focal } \\
\text { Heart (Aorta) }\end{array}$ & 1 & $2 / 12$ & $2 / 12$ & $1 / 12$ & $2 / 12$ \\
\hline $\begin{array}{l}\text { Myocarditis, focal } \\
\text { Pancreas }\end{array}$ & $1-2$ & $2 / 12$ & $0 / 12$ & $1 / 12$ & $0 / 12$ \\
\hline $\begin{array}{l}\text { Focal round cell } \\
\text { collection } \\
\text { Liver }\end{array}$ & 1 & $1 / 12$ & $0 / 12$ & $1 / 12$ & $0 / 12$ \\
\hline $\begin{array}{l}\text { Clear cell, } \\
\text { multifocal }\end{array}$ & 1 & $0 / 12$ & $0 / 12$ & $2 / 12$ & $0 / 12$ \\
\hline $\begin{array}{c}\text { Degeneration/Necrosis, } \\
\text { focal. } \\
\text { Kidneys }\end{array}$ & 1 & $0 / 12$ & $0 / 12$ & $1 / 12$ & $0 / 12$ \\
\hline $\begin{array}{l}\text { Focal round cell } \\
\text { collection, cortex } \\
\text { Cystic }\end{array}$ & 1 & $2 / 12$ & $0 / 12$ & $0 / 12$ & $1 / 12$ \\
\hline $\begin{array}{l}\text { degeneration, } \\
\text { medulla, focal }\end{array}$ & 1 & $1 / 12$ & $0 / 12$ & $0 / 12$ & $0 / 12$ \\
\hline $\begin{array}{l}\text { Mineralization, } \\
\text { tubular lumen, } \\
\text { focal }\end{array}$ & 1 & $0 / 12$ & $1 / 12$ & $0 / 12$ & $2 / 12$ \\
\hline $\begin{array}{l}\text { Prostate } \\
\text { Focal round cell } \\
\text { collection }\end{array}$ & $1-2$ & $5 / 12$ & - & $5 / 12$ & - \\
\hline $\begin{array}{c}\text { Ovaries and } \\
\text { oviduct }\end{array}$ & & & & & \\
\hline $\begin{array}{l}\text { Focal round cell } \\
\text { collection }\end{array}$ & 1 & - & $2 / 12$ & - & $2 / 12$ \\
\hline
\end{tabular}

a Severity grading according to a previous study [20]. $1=$ minimal $(<10 \%), 2=$ mild $(10-39 \%), 3=$ moderate $(40-79 \%), 4=$ marked $(80-100 \%)$;

${ }^{\mathrm{b}}$ Animal numbers with histopathologic findings/Animal numbers examined. 


\section{Discussion}

Recently, medicinal mushrooms have become a popular dietary supplement to promote health [21]. Considering dietary supplements are likely to be used for a long-term duration, it is necessary to perform appropriate preclinical toxicity assessments to predict toxicity and to select a safe dose for human health. According to previous studies, research on L. rhinocerotis bioactivities and toxicities have mainly focused on the naturally occurring sclerotium [22] and may have overlooked mushroom mycelia from submerged liquid fermentation, which ensures a high uniform quantitative biomass production as well as a high biological value [23]. Using principal component analysis, the chemical profiles revealed that the L. rhinocerotis mycelial extracts cultured under different conditions of liquid fermentation were distinct from those of the sclerotium [12]. As L. rhinocerotis from liquid fermentation merits consideration as a potential substitute for the sclerotium, toxicity testing is imperative to identify the types of adverse effects that may occur and to predict exposures in humans that should be without risk.

In our previous study, the genotoxicity [14] and prenatal developmental toxicity [15] were conducted on LRM and showed no significant findings that lead to meaningful interpretation of toxic effect. However, a subchronic toxicity evaluation is necessary as it can provide valuable information about the toxicity of LRM and can provide suitable dose regimens for long-term studies. Hence, for the first time, the present study investigated the potential toxicity of LRM in rats after 13 weeks of repeated oral administration at concentrations of $0,850,1700$, and $3400 \mathrm{mg} / \mathrm{kg} /$ day.

Given that LRM was developed as a health supplement, the amino acid and proximate analyses of LRM were first measured, as they are important indices to classify the nutritional value of a food material [24]. In amino acid analysis, the LRM contained a total of $21,728 \mathrm{mg} / 100 \mathrm{~g}$, with the abundant amino acids being glutamate $(5636 \mathrm{mg})$, aspartate $(2384 \mathrm{mg})$, and arginine $(1766 \mathrm{mg})$. Similar to previously reported L. rhinocerotis sclerotia, all essential amino acids, except for tryptophan, were present [25]. Moreover, earlier studies showed that major constituents of sclerotia were carbohydrates $(82.60 \pm 0.01 \%)$, protein $(7.02 \pm 0.20 \%)$, and fat $(0.49 \pm 0.00 \%)$ with an energy of $362.83 \pm 0.76 \mathrm{kcal} / \mathrm{kg}$ [25]. In this study, the carbohydrate $(79.3 \pm 4.6 \%)$, protein $(8.4 \pm 5.3 \%)$, and fat content $(1.6 \pm 2.2 \%)$ of LRM were comparable to those of the reported sclerotia, providing a basis for considering the mycelium as an alternative to the sclerotium. Further, these values are in a similar range to those reported from a previous study $(73.01 \pm 0.04 \%$ carbohydrates, $7.87 \pm 0.01 \%$ protein, $2.30 \pm 0.06 \%$ fat, and $344.20 \pm 0.71$ energy) [25]. According to the results of a recent clinical trial, a high level of plant-based carbohydrates and low-fat diet for 16 weeks was found to reduce body weight and body fat as well as to improve insulin function in overweight individuals [26]. Considering that LRM has a high level of carbohydrates and very low level of lipids, these findings suggested that LRM could replace sclerotium and fits the nutritional requirement for those who are on a weight-management program.

As some mushrooms are bitter [27], consideration should also be given to the taste of medicinal mushrooms for supplement formulation. Among amino acids, aspartic and glutamic acid are classified as monosodium glutamate-like (MSG-like) components, which give mushroom a peculiar umami or palatable taste. In a previous study, the ratio of umami amino acids to total amino acids (umami index) of various mushrooms ranged between 0.21 and 0.32 [28]. A higher umami index (0.37), however, was found in this study. A possibility is that $L$. rhinocerotis contributes protease and peptidase during fermentation, which increases the formation of these taste-active amino acids [29]. Umami substances via G protein-coupled receptors and heteromeric T1R1 + T1R3 receptors not only could elicit umami taste but also exert many health benefits, including fat regulation [30]. Considering that LRM provides not only rich nutrients, but also gives a sour and umami taste, which contributes a pleasant taste to the food, it thus could be used as a natural source for development and application in food products.

When no human data on LRM are available, animal data are essential for a safety assessment. In this study, treatment with LRM for 13 weeks at up to $3400 \mathrm{mg} / \mathrm{kg} /$ day 
was not associated with adverse effects on toxicologically-relevant parameters in rats of either sex. Although there was a linearly decreased feed intake in both the control and the treatment groups during the study period, no significant differences between the groups were found as compared to the control group, except for the male rats in the high-dose group. These decreases may be explained by the fact that animals regulate their intake according to dietary energy level [31]. Studies have shown that consumption of a high-energy diet could decrease the level of adenosine monophosphate-activated protein kinase (AMPK) expression in the hypothalamus, resulting in reduced hunger and enhanced satiety $[32,33]$. In line with these studies, as the commercial diet and LRM both contained high energy (with 3.42 and $3.34 \mathrm{kcal} / \mathrm{g}$, respectively), the results of this study suggest that rodents can adjust their feed intake according to the energy state of the diet and thereby decreased feed intake.

Despite decreased feed intake, there was no change directly proportional to body weight. Alterations in body weight are sensitive indicators of adverse effects of drugs and chemicals [34]. After 13 weeks of treatment of LRM, all the animals exhibited a normal increment in body weight, suggesting that LRM did not interfere with the normal metabolism of animals. Moreover, there were no significant differences among the treatment groups based on the hematological and serum chemical parameters analyses, indicating that exposure to LRM caused no injury to the hematopoietic, hepatic, cardiac, and renal systems [35]. Further, in urinalysis, there were no treatment-related effects for both sexes in the treatment groups, demonstrating that LRM produces well-functioning excretory systems.

Organ weight changes are indicators of possible morphological or functional changes. In this study, no significant changes in the weight of the brain, heart, kidneys, liver, spleen, or testes were observed. Although ovaries with oviducts weight $(0.14233 \pm 0.02466)$ in the low-dose group were slightly higher than those of the control group $(0.11606 \pm 0.01284)$ after 13 weeks, these changes lack dose-relationship and treatment-related histological findings. Hence, these differences were considered to be normal biological variation.

In the histopathological examination, the lesions found in the $3400 \mathrm{mg} / \mathrm{kg} \mathrm{LRM}$ groups include focal round cell collection (harderian glands, pancreas, kidney, prostate, and ovaries and oviduct), cysts (thyroid), inflammation (lung and heart), degeneration (liver and kidney), and tubular lumen mineralization (kidney), which are common in repeated toxicity studies [20]. However, these lesions were also observed in the control group with similar frequency and severity grade. Considering that these lesions were minor, did not follow a dose-dependent pattern, and within normal ranges in all the animals, it can be concluded that changes might have been background lesions and not a treatment-related effect.

\section{Conclusions}

Based on the findings of this study, dietary administration of LRM to Sprague-Dawley rats over a period of 13 weeks resulted in a no-observed-adverse-effect level (NOAEL) of $3400 \mathrm{mg} / \mathrm{kg} \mathrm{BW} /$ day. To extrapolate the results from rats to humans, a default uncertainty factor of 100 was applied and the ADI for LRM can be estimated as $34 \mathrm{mg} / \mathrm{kg}$ BW/day. Although the findings may be used in the future for reference, initial clinical trials involving healthy adult volunteers are required to assure both the safety and efficacy of LRM.

Author Contributions: Conceptualization, C.-C.C.; Data curation, I.-C.L. and B.-H.Y.; Methodology, J.-Y.L.; Resources, B.-H.Y. and J.-Y.L.; Supervision, C.-C.C.; Validation, S.L.; Writing-original draft, I.-C.L.; Writing-review \& editing, I.-C.L., S.L. and C.-C.C. All authors have read and agreed to the published version of the manuscript.

Funding: This research received no external funding.

Institutional Review Board Statement: All animal procedures were reviewed and approved by the Institutional Animal Care and Use Committee at Level Laboratory Animal Center in Taiwan (approval No.: 120201-04).

Informed Consent Statement: Not applicable. 
Conflicts of Interest: The authors have no conflicts of interest to declare that are relevant to the content of this article.

\section{References}

1. Ulziijargal, E.; Mau, J.L. Nutrient compositions of culinary-medicinal mushroom fruiting bodies and mycelia. Int. J. Med. Mushrooms 2011, 13, 343-349. [CrossRef] [PubMed]

2. Wasser, S.P.; Weis, A.L. Medicinal Properties of Substances Occurring in Higher Basidiomycetes Mushrooms: Current Perspectives (Review). Int. J. Med. Mushrooms 1999, 1, 31-62. [CrossRef]

3. Wasser, S.P. Medicinal mushroom science: Current perspectives, advances, evidences, and challenges. Biomed. J. 2014. [CrossRef] [PubMed]

4. Lee, S.S.; Chang, Y.S.; Noraswati, M.N.R. Utilization of macrofungi by some indigenous communities for food and medicine in Peninsular Malaysia. Forest Ecol. Manag. 2009, 257, 2062-2065. [CrossRef]

5. Wong, K.H.; Cheung, P.C.K. Sclerotia: Emerging Functional Food Derived from Mushrooms. In Mushrooms as Functional Foods; John Wiley\& Sons, Inc.: Hoboken, NJ, USA, 2008; pp. 111-146. [CrossRef]

6. Wong, K.-H.; Lai, C.K.M.; Cheung, P.C.K. Immunomodulatory activities of mushroom sclerotial polysaccharides. Food Hydrocoll. 2011, 25, 150-158. [CrossRef]

7. $\quad$ Eik, L.-F.; Naidu, M.; David, P.; Wong, K.-H.; Tan, Y.-S.; Sabaratnam, V. Lignosus rhinocerus (Cooke) Ryvarden: A Medicinal Mushroom That Stimulates Neurite Outgrowth in PC-12 Cells. Evid. Based Complementary Altern. Med. 2012, 2012, 7. [CrossRef]

8. Lee, M.L.; Tan, N.H.; Fung, S.Y.; Tan, C.S.; Ng, S.T. The Antiproliferative Activity of Sclerotia of Lignosus rhinocerus (Tiger Milk Mushroom). Evid. Based Complement. Alternat. Med. 2012, 2012, 697603. [CrossRef]

9. Lee, S.S.; Tan, N.H.; Fung, S.Y.; Sim, S.M.; Tan, C.S.; Ng, S.T. Anti-inflammatory effect of the sclerotium of Lignosus rhinocerotis (Cooke) Ryvarden, the Tiger Milk mushroom. BMC Complementary Altern. Med. 2014, 14, 359. [CrossRef]

10. Willetts, H.J.; Bullock, S. Developmental biology of sclerotia. Mycol. Res. 1992, 96, 801-816. [CrossRef]

11. Abdullah, N.; Haimi, M.Z.D.; Lau, B.F.; Annuar, M.S.M. Domestication of a wild medicinal sclerotial mushroom, Lignosus rhinocerotis (Cooke) Ryvarden. Ind. Crops Prod. 2013, 47, 256-261. [CrossRef]

12. Lau, B.F.; Abdullah, N.; Aminudin, N.; Lee, H.B.; Yap, K.C.; Sabaratnam, V. The Potential of Mycelium and Culture Broth of Lignosus rhinocerotis as Substitutes for the Naturally Occurring Sclerotium with Regard to Antioxidant Capacity, Cytotoxic Effect, and Low-Molecular-Weight Chemical Constituents. PLoS ONE 2014, 9, e102509. [CrossRef] [PubMed]

13. Yap, H.Y.; Chooi, Y.H.; Firdaus-Raih, M.; Fung, S.Y.; Ng, S.T.; Tan, C.S.; Tan, N.H. The genome of the Tiger Milk mushroom, Lignosus rhinocerotis, provides insights into the genetic basis of its medicinal properties. BMC Genom. 2014, 15, 635. [CrossRef] [PubMed]

14. Chen, T.I.; Zhuang, H.W.; Chiao, Y.C.; Chen, C.C. Mutagenicity and genotoxicity effects of Lignosus rhinocerotis mushroom mycelium. J. Ethnopharmacol. 2013, 149, 70-74. [CrossRef] [PubMed]

15. Jhou, B.-Y.; Liu, H.-H.; Yeh, S.-H.; Chen, C.-C. Oral reproductive and developmental toxicity of Lignosus rhinocerotis mycelium in rat. J. Ethnopharmacol. 2017, 208, 66-71. [CrossRef]

16. Baur, F.J.; Ensminger, L.G. The Association of Official Analytical Chemists (AOAC). J. Am. Oil Chem. Soc. 1977, 54, 171-172. [CrossRef]

17. Fung, S.Y.; Cheong, P.C.H.; Tan, N.H.; Ng, S.T.; Tan, C.S. Nutritional evaluation on Lignosus cameronensis C. S. Tan, a medicinal Polyporaceae. IUBMB Life 2019, 71, 821-826. [CrossRef]

18. OECD. Test No. 408: Repeated Dose 90-Day Oral Toxicity Study in Rodents; OECD Publishing: France, Paris, 2018. [CrossRef]

19. Zhang, Q.; Zeng, S.X.; Lu, H. Determination of Maximum Tolerated Dose and Toxicity of Inauhzin in Mice. Toxicol. Rep. 2015, 2, 546-554. [CrossRef]

20. Shackelford, C.; Long, G.; Wolf, J.; Okerberg, C.; Herbert, R. Qualitative and Quantitative Analysis of Nonneoplastic Lesions in Toxicology Studies. Toxicol. Pathol. 2002, 30, 93-96. [CrossRef]

21. Chang, S.T.; Wasser, S.P. Current and Future Research Trends in Agricultural and Biomedical Applications of Medicinal Mushrooms and Mushroom Products (Review). Int. J. Med. Mushrooms 2018, 20, 1121-1133. [CrossRef]

22. Nallathamby, N.; Phan, C.-W.; Seow, S.L.-S.; Baskaran, A.; Lakshmanan, H.; Abd Malek, S.N.; Sabaratnam, V. A Status Review of the Bioactive Activities of Tiger Milk Mushroom Lignosus rhinocerotis (Cooke) Ryvarden. Front. Pharmacol. 2018, 8. [CrossRef]

23. Papaspyridi, L.-M.; Aligiannis, N.; Topakas, E.; Christakopoulos, P.; Skaltsounis, A.-L.; Fokialakis, N. Submerged Fermentation of the Edible Mushroom Pleurotus ostreatus in a Batch Stirred Tank Bioreactor as a Promising Alternative for the Effective Production of Bioactive Metabolites. Molecules 2012, 17, 2714-2724. [CrossRef] [PubMed]

24. Sousa, E.C.; Uchôa-Thomaz, A.M.A.; Carioca, J.O.B.; Morais, S.M.D.; Lima, A.D.; Martins, C.G.; Alexandrino, C.D.; Ferreira, P.A.T.; Rodrigues, A.L.M.; Rodrigues, S.P.; et al. Chemical composition and bioactive compounds of grape pomace (Vitis vinifera L.), Benitaka variety, grown in the semiarid region of Northeast Brazil. J. Food Sci. Technol. 2014, 34, 135-142. [CrossRef]

25. Lau, B.F.; Abdullah, N.; Aminudin, N. Chemical Composition of the Tiger's Milk Mushroom, Lignosus rhinocerotis (Cooke) Ryvarden, from Different Developmental Stages. J. Agric. Food Chem. 2013, 61, 4890-4897. [CrossRef] [PubMed]

26. Kahleova, H.; Tura, A.; Hill, M.; Holubkov, R.; Barnard, N.D. A Plant-Based Dietary Intervention Improves Beta-Cell Function and Insulin Resistance in Overweight Adults: A 16-Week Randomized Clinical Trial. Nutrients 2018, 10, 189. [CrossRef] [PubMed] 
27. Chun, S.; Chambers, E.; Han, I. Development of a Sensory Flavor Lexicon for Mushrooms and Subsequent Characterization of Fresh and Dried Mushrooms. Foods 2020, 9, 980. [CrossRef] [PubMed]

28. Wang, X.-M.; Zhang, J.; Wu, L.-H.; Zhao, Y.-L.; Li, T.; Li, J.-Q.; Wang, Y.-Z.; Liu, H.-G. A mini-review of chemical composition and nutritional value of edible wild-grown mushroom from China. Food Chem. 2014, 151, 279-285. [CrossRef]

29. Zhao, C.J.; Schieber, A.; Gänzle, M.G. Formation of taste-active amino acids, amino acid derivatives and peptides in food fermentations-A review. Food Res. Int. 2016, 89, 39-47. [CrossRef]

30. Zhang, Y.; Venkitasamy, C.; Pan, Z.; Liu, W.; Zhao, L. Novel Umami Ingredients: Umami Peptides and Their Taste. J. Food Sci. 2017, 82, 16-23. [CrossRef]

31. Sayed, A.-B.N. Effect of different dietary energy levels on the performance and nutrient digestibility of lambs. Vet. World 2009, 2, 418-420.

32. Hu, X.; Wang, Y.; Sheikhahmadi, A.; Li, X.; Buyse, J.; Lin, H.; Song, Z. Effects of dietary energy level on appetite and central adenosine monophosphate-activated protein kinase (AMPK) in broilers. J. Anim. Sci. 2019, 97, 4488-4495. [CrossRef]

33. Moosavian, S.P.; Haghighatdoost, F. Dietary energy density and appetite: A systematic review and meta-analysis of clinical trials. Nutrition 2020, 69, 110551. [CrossRef] [PubMed]

34. Porwal, M.; Khan, N.A.; Maheshwari, K.K. Evaluation of Acute and Subacute Oral Toxicity Induced by Ethanolic Extract of Marsdenia tenacissima Leaves in Experimental Rats. Sci. Pharm. 2017, 85, 29. [CrossRef] [PubMed]

35. Kaid, F.; Alabsi, A.M.; Alafifi, N.; Ali-Saeed, R.; Ameen Al-koshab, M.; Ramanathan, A.; Ali, A.M. Histological, Biochemical, and Hematological Effects of Goniothalamin on Selective Internal Organs of Male Sprague-Dawley Rats. J. Toxicol. 2019, 2019, 6493286. [CrossRef] [PubMed] 